\title{
Mathematical modelling of the within-host dynamics of Plasmodium falciparum
}

\author{
M. B. $\operatorname{HOSHEN}^{1 *}$, R. HEINRICH${ }^{2}$, W. D. STEIN ${ }^{1}$ and H. GINSBURG ${ }^{1}$ \\ ${ }^{1}$ Department of Biological Chemistry, Institute of Life Sciences, The Hebrew University of Ferusalem, \\ Ferusalem 91904, Israel \\ ${ }^{2}$ Institute of Biology, Humboldt University Berlin, Invalidenstrasse 42, D-10115 Berlin, Germany
}

(Received 15 December 1999; revised 16 March 2000; accepted 16 March 2000)

\section{SUMMARY}

The development of malaria due to Plasmodium falciparum is a complex, multi-stage process. It is usually characterized by an exponential growth in the number of parasite-infected erythrocytes, followed by marked oscillations in this number with a period of $48 \mathrm{~h}$, which are eventually dampened. This course of events has been the subject of various mathematical models. In this paper we propose a new mathematical model for the in-host asexual erythrocytic development of $P$. falciparum malaria. Synchronicity of the infection is shown to be an inherent feature of infection, irrespective of the duration of merozoite release from the liver. It will, therefore, cause periodic symptoms, as known in malaria patients. We also simulate the effects of an induced host immune response and show how the level of immunity affects the development of disease. The simulations fit well with the clinical observations. We show how infection can become asynchronous and discuss the effect of desynchronization on the circulating and total parasitaemia and demonstrate that synchronized broods will show parasitaemia fluctuations.

Key words: Plasmodium falciparum, mathematical model, within-host, synchrony.

\section{IN TRODUCTION}

Malaria presents some clear clinical phenomena. One of the most pronounced is parasitaemia in the bloodstream, that causes the clinical symptoms. The parasitaemia initially increases exponentially, then achieves a transient steady state, modulated by strong oscillations with a period of 2 days. These oscillations are eventually damped with or without decline in parasitaemia. During the last few years there has been considerable work on the mathematical modelling of the dynamics of Plasmodium falciparum infections. These studies model at the level of within-host dynamics (either when treated with drugs or else when untreated; White (1997)), involving the onset of host immunity (Antia, Nowak \& Anderson, 1996), or they model the epidemiology, concentrating on patterns of infection and strain selection (Gupta et al. 1994). In some cases the vector-host interaction is also considered (McKenzie $\&$ Bossert, 1997). One of the leading models used is that of Anderson, May \& Gupta (1989). This model attempts to address the blood-stage asexual cycle of $P$. falciparum, by following the invasion of erythrocytes (RBC) by merozoites (M), and the resulting

* Corresponding author: Department of Biological Chemistry, Institute of Life Sciences, Hebrew University of Jerusalem, Jerusalem 91904, Israel.

Tel: +972 2 6585409. Fax: +97226585440.

E-mail: hoshen@vms.huji.ac.il infected red blood cells (IRBC). These IRBCs rupture after $48 \mathrm{~h}$, releasing merozoites into the bloodstream to cause further invasion. Mathematically, one may write 3 coupled differential equations :

$d x / d t=\Lambda-\mu x-\beta x s$

$d y / d t=\beta x s-\alpha y$

$d s / d t=\alpha r y-\delta s-\beta x s$,

with $x$ being the uninfected RBC concentration (per $\mu \mathrm{l}), y$ that of the IRBC and $s$ that of the merozoites. Bone-marrow erythropoiesis is at a fixed rate $\Lambda$, and the normal decay of RBCs is a first-order process with rate constant $\mu$. Merozoites are released from rupturing IRBCs, at rate $\alpha$, with multiplication rate $r$ per cycle. They are eliminated by one of two processes: by invading fresh $\mathrm{RBCs}$ at a rate proportional to both the concentrations of $\mathrm{RBC}$ and merozoites with coefficient $\beta$, or loss of infectivity, culminating with death (first-order with rate constant $\delta)$.

A drawback of this mathematical model is its nonlinearity, which makes analytical solution difficult, and thus dependent on guessing the parameters to fit experimental data. Intuitive approximations cannot be made when in this form.

An additional shortcoming is the inherent lack of synchronicity or periodicity that results from the model. The cause of synchrony of malaria infections, and the well-known resulting periodicity of 
symptoms, is still unclear. Even if one assumes that all merozoites emerge from the liver during a limited time-window, no synchrony of the erythrocytic phase would be expected by this model. Such synchronicity is, however, reported (Boyd \& Kitchen, 1937; Cheng et al. 1997), and peaks were found with typical width of 4-6 h (White, Chapman \& Watt, 1992). In order to explain the clinical features of infection, Kwiatkowski and his colleagues (Kwiatkowski \& Nowak, 1991; Gravenor \& Kwiatkowski, 1998a) suggested a synchronizing mechanism in order to compensate for the inherent lack of synchrony in the Anderson et al. (1989) model. In their model, Kwiatkowski and colleagues relate synchrony to fever. According to their proposal, high parasitaemia, or more precisely, large synchronized bursting or infected erythrocytes, induces fever in the host. This may have to do with release of pyrogens from the IRBCs. They picture this as a modulation of the stage-specific survival rate of the parasites, and model it by a coupled Markov chain process (Gravenor \& Kwiatkowski, 1998a). This mechanism would be induced by bursting schizonts but would not harm the tiny-ring stage, thus not endangering the inducing population. However, Cheng et al. (1997) demonstrated that synchrony exists even at subsymptomatic parasitaemia, that is, in the absence of fever. Molineaux \& Dietz (1999) reviewed and criticized the recent models, but did not present their own solution.

Another model, which is mainly phenomenological, is that of White et al. (1992), which relates to a single stage, the infected $\mathrm{RBC}$, and a per-cycle multiplication rate. Drug interaction is treated in this model by a parasite reduction ratio, which is the per-cycle reduction rate of parasite number under drug pressure (White, 1997).

It would be beneficial to combine the two methods of White et al. and Anderson et al. to form a single theory which can explain the periodicity of malaria solely through the 3-compartment (RBC, IRBC and merozoites) approach, and which would enable a mathematical basis for analysis of immunity and chemotherapy.

\section{METHODOLOGY}

We would like to discuss some facets of the basic Anderson et al. (1989) model, in order to modify some points, which may provide a basis for immunity and vaccination/chemotherapeutic modelling. A possible objection to the basic model is the use of first-order processes to represent processes completed at a fixed time. The life-cycle of the intraerythrocytic parasite is $\tau=48 \mathrm{~h}$. Only under constant parasitaemia conditions of unsynchronized infection, would it be acceptable to claim that $1 / 48$ of the IRBCs burst every hour. Obviously, only those of between 47 and $48 \mathrm{~h}$, and all of them, will burst in the next hour, independently of the populations of other stages. This point is the centre of the correspondence between Saul (1998) and Gravenor \& Lloyd (1998b). The resolution of this discussion was far from satisfactory. Saul suggested using either a false multiplication rate, or else working in a discrete-time model. Gravenor and Lloyd's solution was to form $n$ compartments, which would represent the various stages of parasite development. Even under this approximation, as used by these authors (Gravenor et al. 1998a,b), rates are still first-order, meaning that a single parasite has a finite probability of remaining for $48 \mathrm{~h}$ at its initial ring-stage. This form of cycle-arrest is found neither in infected patients nor in the laboratory, in the absence of cytostatic drugs. An additional drawback of that model is that it is unsuitable for analytical or semianalytical calculations.

For simulation purposes, this approach could have been modified, by splitting the population into stages of 1-h 'boxes'. Each box (say box $n$ ) would simulate the population aged between $n-1$ and $n$ h. The whole population of each box moves to the next box at each simulated 'hour', after the subtraction of the suitable death rates proposed. Thus a discrete growth model with a continuous first-order kill-rate could be formed that would guarantee a full $48 \mathrm{~h}$ cycle, while allowing detailed stage-specific analysis of drug and immune interactions. We feel, however, that even this useful amendment (which we shall ourselves use later in this paper), is not the ideal one for the issue at hand, the modification of the full 3element model.

\section{The proposed model}

We shall now present the foundations for a novel reformulation of the basic model. The proposed model explicitly introduces a parameter $\tau$, as the life-span of the IRBC, equal to $48 \mathrm{~h}$ in falciparum malaria. The differential equations may now be restated. The only cause for increase of the IRBC population at time $t$ is the instantaneous invasion of RBCs, dependent on $s(t)$. The causes of decreasing IRBC numbers are 2-fold: (1) the invasion $48 \mathrm{~h}$ ago (dependent on $s(t-\tau)$ ), which determines the rate of IRBC bursting at present-time $t$; (2) the decay rate of IRBCs, as a result of spontaneous destruction, host immunity, chemotherapy and gametocyte formation. Similarly, the merozoite population, after the initial release from the liver, increases only by release from bursting IRBCs, which is determined by the size of the merozoite population $48 \mathrm{~h}$ previously. Depletion of merozoite numbers arises either from invasion, or else from ageing or opsonization with anti-merozoite antibodies, which causes a loss in the capability for further invasion. Thus far we may write the equations as a coupled 


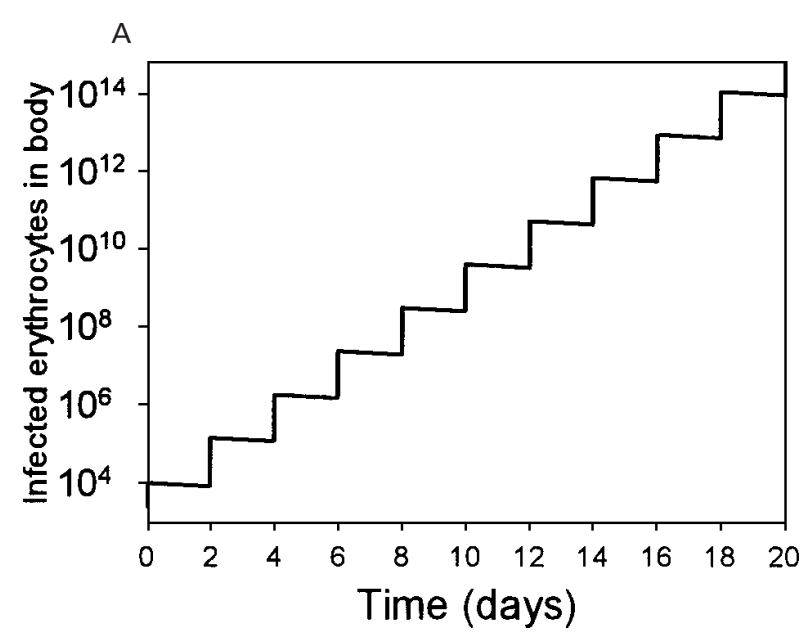

B
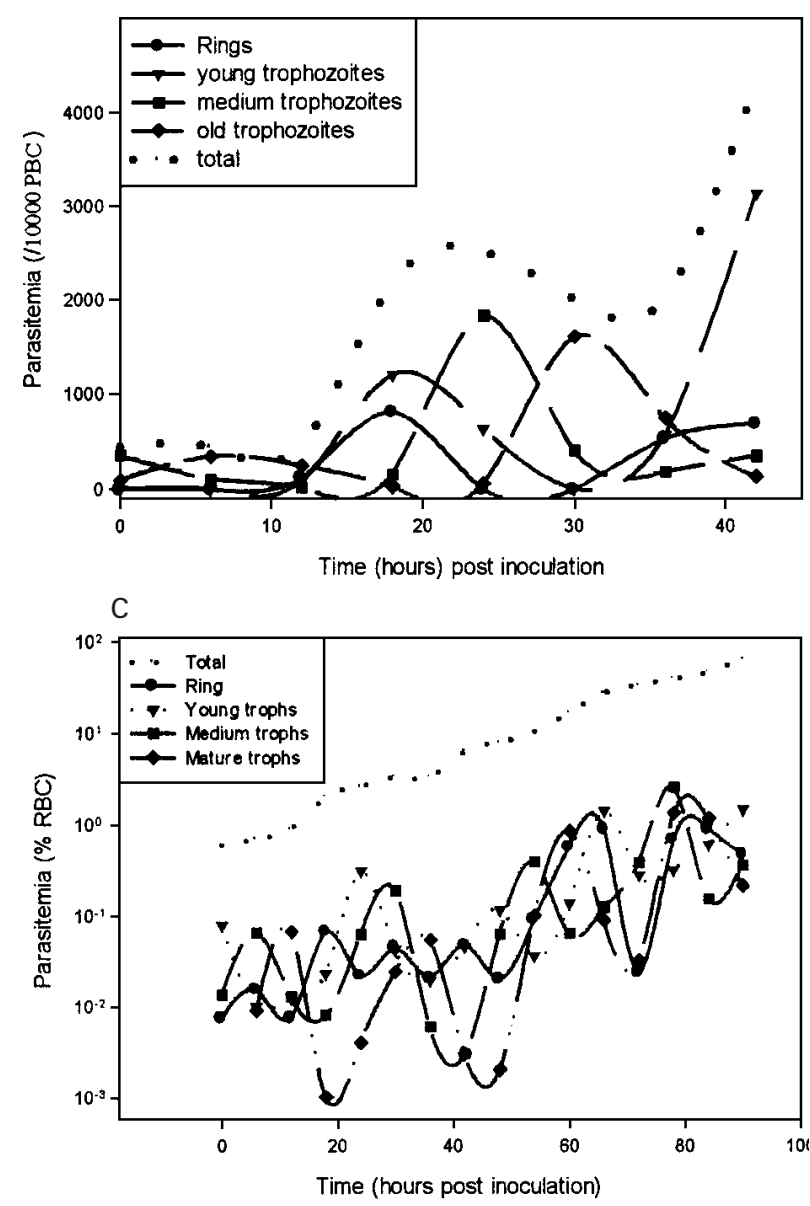

Fig. 1. Time-course of parasitaemia progression. (A) Analytical solution, according to Equations (2) and (3) for the time-course of parasitaemia progression, after an initial release of $10^{4}$ merozoites into the bloodstream at time $t=0$ days. $\alpha=0 \cdot 1 /$ day, $\beta=70 /$ day, $r=16, \delta=0$. Ordinate, number of infected erythrocytes in body; abscissa, time in days from release of merozoites from liver. (B) Data from Caillard et al. (1992). Ordinate, number of Plasmodium vinckei petteri parasites counted at each stage (single mouse). Abscissa, time in hours from inoculation. Total parasitaemia (……). (C) Data from Deharo (1995). Ordinate: average (5 mice) percentage of RBC parasitized with $P$. chabaudi chabaudi. Abscissa, time in hours from inoculation. system of nonlinear differential equations with timedelay:

$$
\begin{aligned}
& d x / d t=\Lambda-\mu x-\beta x s(t) \\
& d y / d t=\beta x s(t)-\beta^{*} x s(t-\tau)-\alpha y \\
& d s / d t=r \beta^{*} x s(t-\tau)-\delta s(t)-\beta x s(t),
\end{aligned}
$$

with $\tau$ the period (for $P$. falciparum $48 \mathrm{~h}$ ), and $\beta^{*}=$ $\beta \mathrm{e}^{-\alpha \tau}$ that part of the merozoite invasion $48 \mathrm{~h}$ ago, which survived a full cycle within IRBC. The equations are, however, still non-linear and do not avail themselves readily to analytical solution.

There is, however, another point that has been overlooked in previous models. When merozoites are released into the bloodstream, their chance of meeting an uninfected RBC is tremendously high. Even during hyperanaemia, with a haematocrit of $30-40 \%$, the distance to the nearest RBC is less than the radius of a $\mathrm{RBC}$. Also, both $\mathrm{RBC}$ and the merozoites are in motion. Thus, they will meet, in the time-scale of interest, almost immediately. Whether or not, the merozoites present at the right orientation to the surface of the erythrocyte or not (Bannister \& Dluzewski, 1990) and the effectivity of the cytoadherence will set the $\beta$ factor. The $x$ term (healthy RBC population) in the merozoite equation would thus be irrelevant except for very extreme RBC depletion, as could be the case in severe malaria. It is more likely that ineffective adherence to IRBC (which are infected already) will be of more importance than is $x$, the RBC concentration. Also, with increasing disease, both a repressed erythropoiesis and spleen function will be relevant, thus reducing the applicability of the basic models. Total obstruction of post-capillary venules by sequestered IRBCs, as observed in cerebral malaria (Silamut et al. 1999), would also reduce the infectivity of merozoites when parasitaemia is high. However, in the case of non-severe malaria considered here, we could forgo the dependency on the number of uninfected erythrocytes.

The resulting equations are then:

$d x / d t=\Lambda-\mu x-\beta s(t) ;$

$d y / d t=\beta s(t)-\beta^{*} s(t-\tau)-\alpha y$

$d s / d t=r \beta^{*} s(t-\tau)-\delta s(t)-\beta s(t)$.

Note that $\beta$ and $\beta^{*}$ are now true rate constants. One may comment that the intrinsic clearance of RBC is also not a first-order process, but is highly preferential for older erythrocytes. The result of a correction here (in Equation 1 a) would be that, with increased parasitaemia, the intrinsic factor $\mu x$ would increasingly be an overestimate, as few RBCs would ever reach that stage.

Total parasitaemia (……). Note: for rodents $\tau=24 \mathrm{~h}$, for human $P$. falciparum, $\tau=48 \mathrm{~h}$. 
RESULTS AND DISCUSSION

\section{Analytical solution for merozoite and erythrocytic- stage populations}

Biologically, the equations now have greater meaning. Mathematically, we have succeeded in separating the variables, by biological analysis. Also, the equations are now linear, which means that the superimposing of subpopulations is permitted. The third equation may be solved analytically as a delayed differential equation (DDE).

This may be done either by a Laplace transform or by piecewise integration, and substituting boundary conditions at each stage. As this method is not well known, we describe it in detail in the Appendix. There we demonstrate that the merozoite population is, as a function of time, during the $n$th parasite lifecycle:

$s(t) \approx s_{0} \mathrm{e}^{-(\beta+\delta)(t-n \tau)} \frac{(\beta \sigma)^{n}}{n !}(t-n \tau)^{n}$.

Here we write $\sigma=r \mathrm{e}^{-\alpha \tau}$, as the effective per cycle multiplication rate (the number of merozoites formed by 1 successful invader). Integration for $y(t)$ is possible, also presented in the Appendix:

$y(t)=\left[y(\tau)+s_{0} \beta\left(r^{n}\left(\frac{\beta}{\beta+\delta-\alpha}\right)^{n}-1\right)\right] \mathrm{e}^{-\alpha t}$,

where $(t>\tau)$ and $y(t)=\left(\mathrm{e}^{-\alpha t}-\mathrm{e}^{-(\beta+\delta) t}\right) \beta s_{0} /(\beta+\delta-\alpha)$ for $0<t \leqslant \tau$. The simulation of Equation (3) yields a population dynamics depicted in Fig. $1 \mathrm{~A}$.

\section{Comparison with experimental data}

This stepped-curve result is obviously different from what is seen in the clinic. This is a result of 2 reasons. The sharp steps result from the assumption of a single merozoite, or sharply synchronized brood, being released initially. The absence of a clear cyclical drop in parasitaemia is a result of our presenting the total parasitaemia, while in vivo for human $P$. falciparum we would measure only the circulating parasitaemia i.e. ring-stage parasites. The influence of this partial representation is discussed by White et al. (1992), who used a discrete-time model, with a single stage of the IRBC, for the progress of the IRBC population. That model finds the well-known rhythmic rise and fall of parasitaemia in human blood. We indeed expect to find the stepform of continuous growth in infections in which there is no sequestration, such as in rodent $P$. vinckei petteri. This is the case, as we can see from the total parasitaemia in the rodent depicted by a dotted line in Fig. 1 B (data adapted from Caillard et al. 1992). In this figure we observe the rise and disappearance of individual stages (heavy lines with symbols), as the broods progress from stage to stage. The periodicity is striking (note that in rodents $\tau=24 \mathrm{~h}$ ).
However, the total parasitaemia rises now steeply at schizogony, with a slight decline between rises. Note also, how in P. chabaudi chabaudi (top line in Fig. 1C, data from Deharo (1995)), loss of initial synchrony (infection was caused by a single inoculation) caused the initial stepping curve to become a plain constant rise. Sequestration of mature IRBC in the vascular endothelium, with the consequent inability to observe trophozoites and schizonts in humans is parallel to observing only the ring stage in the rodent model. We thus see in humans clear periodic rises and falls.

Probably the most detailed descriptions of the evolution of human infection were obtained from the practice of malariotherapy (Boyd \& Kitchen, 1937). The development of infection described in these infections was characterized by an initial exponential increase in parasitaemia, followed by the achievement of a transient steady-state, modulated by strong oscillations with a period of 2 days. These oscillations were eventually damped with or without decline in parasitaemia. A test of our model would be the simulation of these characteristics. We have explained the rise and the oscillations. We must now explain the achievement of steady-state, and the damping of the oscillations.

\section{Immunity and onset of steady state}

Host immunity generally begins a few cycles after the initiation of the intra-erythrocytic phase. It may be reflected by either (1) an increased death rate of IRBC (reflected by $\alpha$ ), caused by macrophage attack on signalled erythrocytes, or else it may involve a protection against the re-invading merozoites. This protection may be either $(2 \mathrm{a})$ by protecting the $\mathrm{RBC}$ (a reduction in $\beta$ ), or, more likely, $(2 \mathrm{~b})$ by incapacitating free merozoites in the bloodstream (reflected by an increase in $\delta$ ). The onset of immunity may be represented mathematically by a trigger mechanism (dependent on time and/or on pathogen concentration), or else by modifying the differential equations in such a way that the parameters $\alpha$ and/or $\beta$ and/or $\delta$ become dependent on the concentration of antibodies. This concentration, at a first approximation, can be assumed to be proportional to the concentration of pathogen. For changing conditions, the analytical solution in equations 2 and 3 is unsuitable, and so we must revert to numerical integration of the differential equations $1(b)$ and 1(c). For this purpose we use an adaptation of the Runge-Kutta method for delay equations. Assuming that the host develops an immune response a week after the initial release of merozoites into the bloodstream (Druilhe \& Perignon, 1994), and assuming that the response is not overwhelming, but only constrains the parasites to steady-state $(d s / d t=0, s(t)=s(t-\tau))$, we can calculate the valid $\delta$, if immunity is lethal to merozoites: $\delta=r \beta^{*}-\beta$. If 


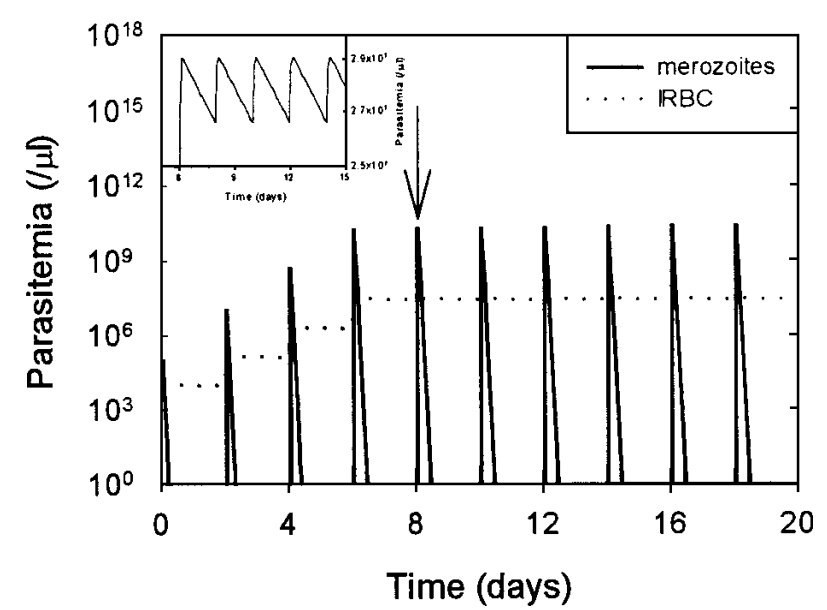

Fig. 2. Effect of immunity of the progression of infection. Simulation by numerical integration of equations $1(\mathrm{a}-\mathrm{c})$, by Runge-Kutta fourth-order method of the time-course of parasitaemia progression, after the initial release of $10^{4}$ merozoites into the bloodstream at time $t=0$ days. Immunity is assumed to set in at time $t=8$ days. $\alpha=0.05 /$ day, $r=16$, initially (before onset of immunity) $\delta=\beta$. Ordinate, parasites in body; abscissa, time in days from release. IRBC (........) and merozoites (-) as a function of time. $\beta=70 /$ day. $\delta=\delta_{s s}=r \beta \mathrm{e}^{-\alpha \tau}-\beta$. (Inset) Blow-up of the IRBC population at and after the onset of immunity in A (note linear scale).

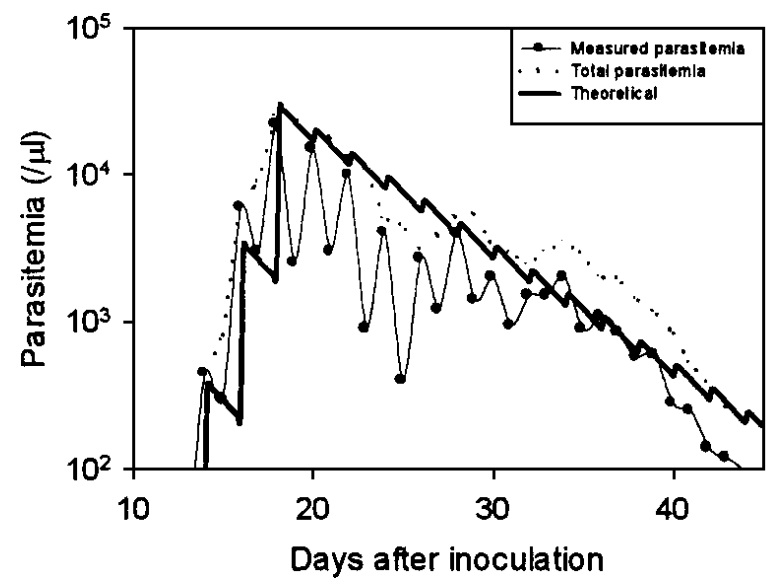

Fig. 3. Time-course of infection in an infected patient. Clinical data of time-course of parasitaemia for malariotherapy-motivated induced malaria in a single patient (data from ref. 7, Fig. 3). Ordinate, IRBC count $/ \mu 1$. Abscissa, time in days from inoculation. $(\bullet-\bullet)$ Measured parasite count. (……) Sum of 2 consecutive measurement of parasitaemia. ( - ) Simulation by theoretical model. $r=16, \alpha=0 \cdot 3$, $\beta=70 /$ day, $\delta=800 /$ day (after onset of immunity).

the mechanism of immunity is not merozoiticidal, but trophozoiticidal i.e. acting on IRBC harbouring trophozoites, it would mean that $\alpha$ is modulated. For steady-state in the absence of considerable $\delta, \alpha=$ $\ln (r) / \tau$. Thus we would expect, if such a response was attained, a check on the growth of parasitaemia which would revert immediately to steady state (Fig. 2 ). Thus initially we see exponential growth of the
IRBC population, only to be checked by the immune response. If $\alpha$ does not happen to be at the steadystate value, parasitaemia will either increase exponentially or decrease eventually.

Notice that small oscillations are still visible in the total parasitaemia (see linear plot Fig. 2 inset). As the infection was initially synchronous, it would stay so. These oscillations would be considerably amplified in clinical measurements as only ring-stage parasites are seen in circulating blood. Thus we would find the strong oscillations depicted by Gravenor et al. (1998a) and by Cheng et al. (1997) and one case reproduced here in Fig. 3 (solid line), as the parasites undergo sequestration and release. These oscillations would continue until loss of synchrony (see below). In Fig. 3 we roughly estimated also the assumed total parasitaemia as the sum of measured parasitaemia on consecutive days (adding to the parasitaemia of each day the parasitaemia measured the day before, dotted line). This line seems to reveal very small oscillations, if at all, as is predicted by our model. In contrast, an asynchronous model such as that of Anderson et al. (1989) cannot explain the oscillations observed in the clinic. Our model's simulation, with an immune response triggered at 20 days after inoculation, is superimposed (heavy solid line in Fig. 3).

If the immune response (as determined by $\delta$ in equations $1 \mathrm{a}-\mathrm{c}$ ) is not modulated correctly by the parasites, the parasitaemia would either continue to increase until death of the hosts (and thus of the parasite population; dotted line in Fig. 4A) or else it would continue to decrease till elimination (dashed line; Fig. 4A), instead of staying constant (solid line, Fig. 4A). There must be some feedback mechanism to cause such modulation. This is probably the case in the premunition seen in native populations of holoendemic areas, in which steady-state is attained. Of course re-infection may also contribute to the maintenance of this steady-state. In Fig. 3 we do indeed observe a case of over-quenching of the parasitaemia. In other cases, the immunity was just sufficient to keep an effective steady-state (see Fig. 1 in Gravenor et al. 1998a). Whereas small changes in $\delta$ have a significant effect on the course of parasitaemia, similar changes in the value of $\beta$ have only a minor effect, suggesting that the precise value of this term is not too important. In Fig. $4 \mathrm{~B}$ we varied $\beta$ between values of 30 and 200, and the difference in parasitaemia was negligible.

\section{Synchrony of parasites}

Need synchrony be created in the host, or is it inherent? If only a single sporozoite invaded the liver as shown experimentally (Cheng et al. 1997), and this sporozoite multiplied by sharply timed division, synchrony would be the rule, not the exception, and would not have to be explained. 
A

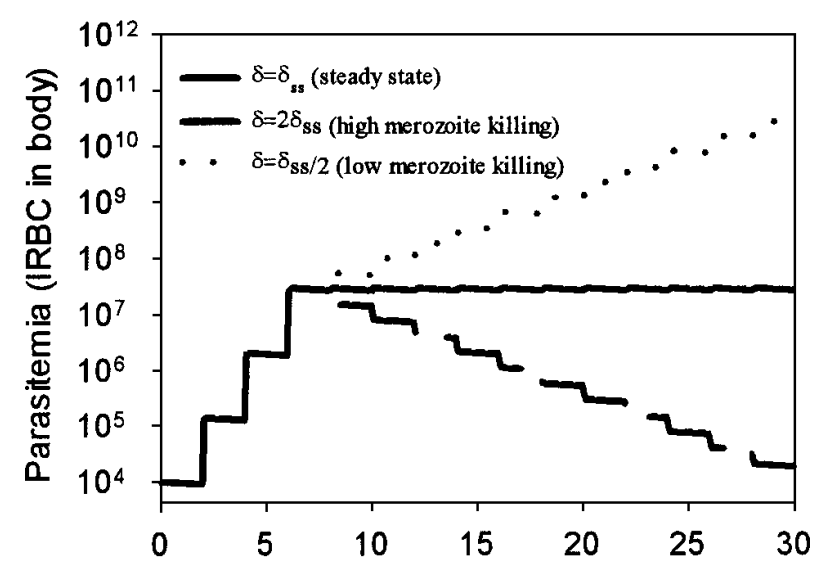

$\mathrm{B}$

Time (days) from initial release from liver

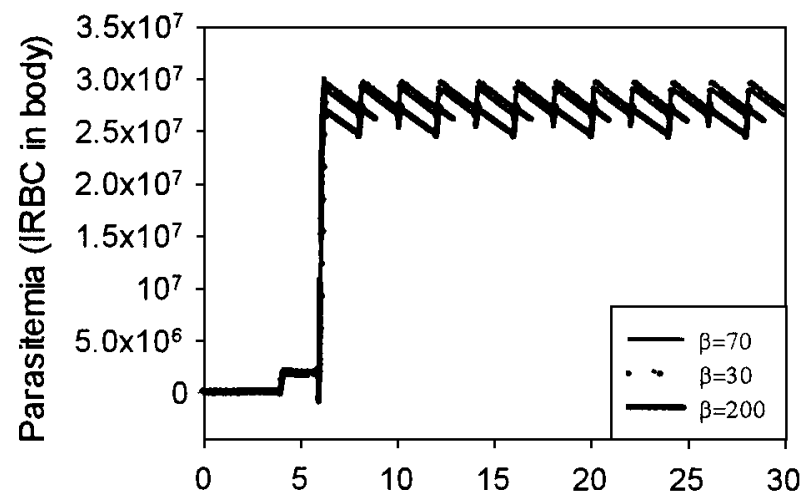

Time(days) from initial release from liver

Fig. 4. Effect of immunity parameters on parasitaemia. Simulation of progress of total parasitaemia, based on numerical integration of equations $(1 \mathrm{~b})$ and $(\mathrm{c})$ as a function of time, changing immunity parameters. Immunity is assumed to set in at time $t=8$ days. $\alpha=0.05 /$ day, $r=16$, initially (before onset of immunity) $\delta=\beta$. (A) Influence of higher or lower merozoite-kill rates, after onset of immune response. $(-) \delta=\delta_{s s}=r \beta \mathrm{e}^{-\alpha \tau}-\beta ;(---) \delta=2 \delta_{s s} ;(\cdots \cdots \cdots)$ $\delta=0 \cdot 5 \delta_{s s}$. (B) Influence of higher or lower invasion rate. $\delta$ set each time to induce steady state. $\beta=70 /$ day $(-)$; $\beta=30 /$ day $(\cdots \cdots \cdots \cdot) ; \beta=200 /$ day $(-)$.

Therefore, the synchronizing mechanism suggested by Kwiatokwski et al. (1991) would be unnecessary. But would synchrony (that is rhythmic fluctuations in the bloodstream parasitaemia) be expected from an asynchronous release of merozoites from the liver? To simulate this case we assume a simple case, which does not require the full mathematical development above. Using the linearity of equations $1(\mathrm{a}-\mathrm{c})$, we may follow each of many subpopulations independently of the others. Thus, we may follow an initially spread-out population, after we have solved the equations for each brood individually. At this stage we may use a 'box'-type simulation of the parasitaemia as function of time (see Methodology section above). Here we assume that parasites are released over the first half of the parasite cycle (Fig.
A
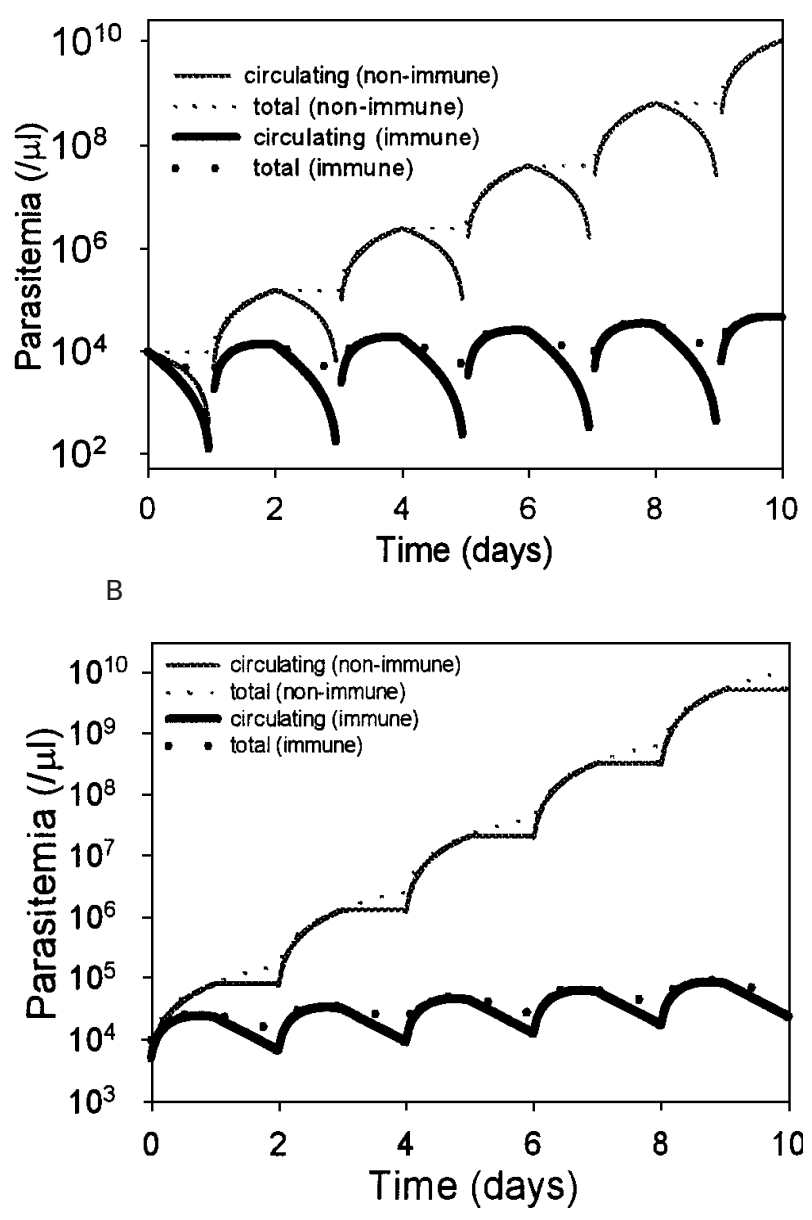

Fig. 5. Effect of timing of liver merozoites release on the evolution of infection. Simulation of time-course of parasitaemia with and without immune response. $10^{4}$ IRBC released at time $t=0$. Ordinate, parasites in body; abscissa time in days. $r=16, \delta=0$. Lines, circulating (ages $0-24 \mathrm{~h}$ ) IRBC. Dots, total IRBC. Light lines and dots, $\alpha=0$ (no immune response). Heavy lines and dots, $\alpha=0 \cdot 05 / \mathrm{h}$. (A) IRBC initial population spread evenly over circulating stages $\left(10^{4} / 24\right.$ in each of $24 \mathrm{~h}$ stages). (B) As (A) initial population spread over all stages (circulating and sequestered $10^{4} / 48$ in each of $48 \mathrm{~h}$ stages). At each hour $(1-\alpha)$ of stage are moved to next stage (and the remainder is removed). Last (number 48) stage is multiplied by 16 and placed in stage 1 .

$5 \mathrm{~A}$ ), or over the whole cycle (Fig. $5 \mathrm{~B}$ ) at uniform density. We also assume 16 -fold multiplication rate per cycle and no killing (lighter lines). We then allow numerical 'progress' of parasites from stage to stage, and follow the total simulated parasitaemia (dotted lines) and the measurable (circulating rings $0-24 \mathrm{~h}$ of age; thin solid lines) parasitaemia with time.

In neither case do we see periodic fluctuations in total parasitaemia. If we assume, however, a constant kill-rate (even stage independent; here $\alpha=0 \cdot 05 / \mathrm{h}$; heavy lines), we do indeed observe large fluctuations in Fig. $5 \mathrm{~A}$ and B, even in total parasitaemia. Such a kill rate could be a result of an immune response. 
Unlike the mechanism suggested by Kwiatkowski and coworkers, here there is no need to invoke a stage-dependent killing.

Thus periodicity is the rule in vivo. Why does desynchronization set in (Boyd \& Kitchen (1937), Fig. 4, and compare Fig. 2 in Cheng et al. (1997) where such a phenomenon is not predicted)? We propose that this process is a result of the stochastic fluctuations in the individual periodicity of the parasite stage-cycle. We must then clarify whether the fluctuation in length of the cycle is genetically determined, or random. By genetically determined, we mean that the length of the life-cycle is a characteristic of the founder of the brood, and thus that the peaks of the different broods would become separated as time progresses. Thus, if genotypical, the standard deviation of the fluctuation of the timing of peaks would increase linearly with time, and desynchronization would be swift. If the fluctuations were random, the length of the life-cycle of the offspring being independent of that of the parent, desynchronization would be slower, being proportional to the square root of time (see Appendix). Thus synchrony would last longer. To depict these two cases we present 2 simulations of wave-packets of sine populations, whose initial standard deviation of periods is fixed at $3 \mathrm{~h}$ (White et al. 1992). We see (Fig. 6 solid line), that when period length is genetic, synchrony is lost quickly. However, with random fluctuations (Fig. 6 dotted line), noticeable synchrony persists longer. Inspection of clinical data indicates that synchronicity can be lost within 6 cycles, favouring the genetically dependent regulation hypothesis. Synchrony, however, can be lost even faster in in vitro cultures, indicating that there may be some cueing mechanism in vivo to instruct the cells to divide together. In principle, synchronization may fulfil several goals. One reason may be the destruction of rival cohorts (by fever, cytokines, obstruction etc.), which, if from other sources, would not be synchronized. Another reason may be minimizing the risk to merozoites by host defences: the well-synchronized efflux of merozoites will swamp the potential B cell mechanisms, reducing the risk to the unprotected merozoites. Thirdly, there might also be a biological benefit given the phenomenon of sequestration. Merozoites would have to (1) try to be the first, in order to get their pick of uninfected RBC, while late arrivers may adhere to infected ones; (2) try to be the last, so that sequestrating parasites do not obstruct the free passage of merozoites and fresh erythrocytes in postcapillary venules. Only good timing would resolve the dilemma. We would thus expect that the synchronization would be activated as a trigger mechanism only at very high parasitaemia. This is not supported by the data. Synchronization is pronounced even at low parasitaemia, and with increasing parasitaemia, thus not supporting the claim that it regulates the total population. Synchronization is also very pronounced in $P$. vivax, which is not as lethal to the host, and does not achieve high parasitaemia. In addition, high spikes of fever are sometimes disastrous also to the inducing cohort. However, the clinical finding that high fever precedes spikes of the circulating parasitaemia curve (cf. Cheng et al. (1997)) suggests that this may indeed be a cueing mechanism. Thus, we would expect a non-destructive cueing mechanism. We have indeed found that also in vitro some cueing may exist, and can be detected by the correlation between the frequency of change of medium and loss of synchrony (Atamna \& Ginsburg, unpublished results). This will require further research.

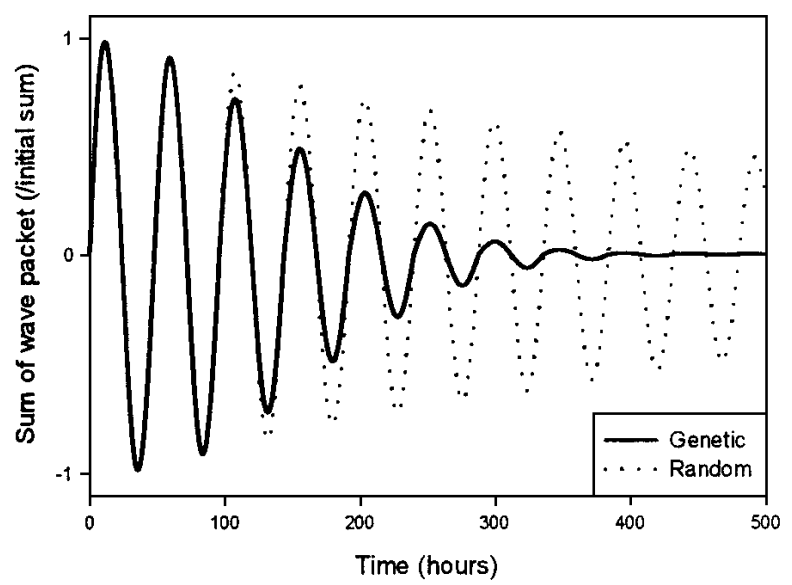

Fig. 6. Loss of synchrony of wave-packets. Sum of wave packet of sine waves, with average period $(\tau) 48 \mathrm{~h}$. Ordinate, sum of waves. Abscissa, time $(t)$ in days. Initial standard deviation $(\sigma) 3 \mathrm{~h} . k=\operatorname{int}(t / \tau)$. $\eta$ fluctuation of period from average. (-) 'genetically determined': $\left.\int_{-\infty}^{\infty} \mathrm{e}^{-\eta^{2} / 2 \sigma^{2}} \sin (t-k \tau-k \eta) d \eta\right)$. (……) Random fluctuation of period:

$\int_{-\infty}^{\infty} \mathrm{e}^{-\eta^{2} / 2 \sigma^{2}} \sin (t-k \tau-\sqrt{ } k \eta) d \eta$.

A P PENDIX

\section{Mathematical solution of modified differential equation}

In the text we attained the following delay differential equation (DDE) for the merozoite population (1 c) $: d s / d t=r \beta^{*} s(t-\tau)-\delta s(t)-\beta s(t)$.

We shall solve this DDE as follows. We shall assume an initial population of $s_{0}$, which at time $t=0$ emerged from the liver. For the first period $0<t<\tau$, the first term contributes nothing. Thus, $d s / d t=-\delta s(t)-\beta s(t)$, as there are no mature IRBCs, none rupture and no merozoites are released. Hence, the merozoite population is diminishing with rate constant $-(\beta+\delta)$, and throughout the first cycle the population is $s(t)=s_{0} \mathrm{e}^{-(\beta+\delta) t}$; and at the end of the cycle $s(\tau)=s_{0} \mathrm{e}^{-(\beta+\delta) \tau}$.

Now we solve the differential equation for $\tau<t<2 \tau$ :

$d s / d t=r \beta^{*} s_{0} \mathrm{e}^{-(\beta+\delta)(t-\tau)}-\delta s(t)-\beta s(t) ;$

with initial condition $s(\tau)=s_{0} \mathrm{e}^{-(\beta+\delta) \tau}$. 
We find that for this stretch

$s(t)=s_{0} \mathrm{e}^{-(\beta+\delta) t}+r \beta^{*}(t-\tau) s_{0} \mathrm{e}^{-(\beta+\delta)(t-\tau)} ;$

with $s(2 \tau)=s_{0} \mathrm{e}^{-(\beta+\delta) 2 \tau}+r \beta^{*} \tau s_{0} \mathrm{e}^{-(\beta+\delta) \tau}$.

Once again this will be used for the next stage etc., and we continue for consecutive stages.

By continuing this process and analysing the stage-by-stage results, one may show by induction that for the $n$th stage $n \tau<t<(n+1) \tau$ :

$s(t)=s_{0} \mathrm{e}^{-(\beta+\delta) t} \sum_{j=0}^{n} \frac{\left(r \beta^{*}\right)^{j}}{j !}(t-j \tau)^{j} \mathrm{e}^{j(\beta+\delta) \tau}$.

This analytical solution is, as commented above, correct while immunity has not changed, and when the constants may be regarded unchanged (capillaries unblocked etc.). Chemotherapy will be reflected as a modification of the IRBC kill rate constant $\alpha$, that is, of $\beta^{*}$. Immunization (natural or by vaccination) is represented either by a decrease in $\beta^{*}$, or else by an increase in the merozoite kill rate constant $\delta$, as above.

What do we know about the rate-constant parameters hereby introduced? The merozoite kill rate, $\delta$ reflects a very swift process. Merozoites are not observed in vivo; thus they must have a life-span of not longer than, say, $20 \mathrm{~min}$. Thus $\delta=72 /$ day will not be an over-estimate (Anderson et al. 1989). Since, invasion is an important process, $\beta$ must be at least similar to $\delta$, and probably larger, so that the merozoites will not be depleted, before infection. As $\alpha$ reflects as slow process, being of relevance to a period of 2 days, $\alpha \ll \delta$, $\beta$. By a similar consideration, as $\mu$ relates to the RBC life-period of 120 days, $\mu \ll \alpha$. The number of merozoites produced per rupturing IRBC, $r$, is known to be 16 per cycle (Cheng et al. 1997).

By these means we can separate the solution for $s(t)$, and subsequently for $y(t)$ and $x(t)$ into timescales. The merozoites vanish very quickly, during each time stretch, only to be replenished at time $\tau$ later. In order to integrate the equation for $y(t)$ we must analyse in more detail our solution for $s(t)$. Of course it would be laborious, and probably ineffective, to integrate the series. We note, however, that by inserting the first exponential into the sum and multiplying the two exponentials, we obtain a sum of terms including $\mathrm{e}^{-(\beta+\delta)(t-j \tau)}$. This term will be vanishingly small except for maximal $j$, that is $j=n$. Thus, for biologically relevant cases in which $n$ is not large, we may write:

$s(t) \approx s_{0} \mathrm{e}^{-(\beta+\delta)(t-n \tau)} \frac{(\beta \sigma)^{n}}{n !}(t-n \tau)^{n}$.

Here we write $\sigma=r \mathrm{e}^{-\alpha \tau}$, as the effective per cycle multiplication rate (the number of merozoites formed by 1 successful invader). Integration for $y(t)$ is now straightforward, as for $t>\tau$ $s(t-\tau) \approx s_{0} \mathrm{e}^{-(\beta+\delta)(t-n \tau)} \frac{(\beta \sigma)^{n-1}}{(n-1) !}(t-n \tau)^{n-1}$.

Or

$s(t) \approx s(t-\tau) \frac{\beta \sigma}{n}(t-n \tau)$.

We now substitute these terms into the $y(t)$ equation. We solve the resulting equation by solving the homogenous differential equation for $y(t): d y / d t=$ $-\alpha y$, which gives of course $y=A \mathrm{e}^{-\alpha t}$ ( $A$ is a constant equal to $y(0))$, and then allow $A=A(t)$, and solve for $A(t)=y(t)^{\alpha t}$ to enable the solution of the inhomogeneous equation. This method is known as variation of the integration constants. This equation now reads:

$\frac{d A}{d t} \mathrm{e}^{-\alpha t}-\alpha A \mathrm{e}^{-\alpha t}=\beta s(t)-\beta * s(t-\tau)-\alpha A \mathrm{e}^{-\alpha t}$,

which may be readily simplified to:

$\frac{d A}{d t}=\mathrm{e}^{\alpha t}\left(\beta s(t)-\beta^{*} s(t-\tau)\right)$.

This must now be integrated. Of course, each term may be integrated independently:

$$
\begin{aligned}
A(t) \approx & A(n \tau)+s_{0} \frac{(\beta r)^{n}}{n !} \beta \int_{n \tau}^{t} \mathrm{e}^{-(\beta+\delta-\alpha)\left(t^{\prime}-n \tau\right)}\left(t^{\prime}-n \tau\right)^{n} d t^{\prime} \\
& -s_{0} \frac{(\beta r)^{n-1}}{(n-1) !} \beta \int_{n \tau}^{t} \mathrm{e}^{-(\beta+\delta-\alpha)\left(t^{\prime}-n \tau\right)}\left(t^{\prime}-n \tau\right)^{n-1} d t^{\prime}
\end{aligned}
$$

And thus:

$$
\begin{aligned}
A(t) \approx & A(n \tau)+s_{0} \frac{(\beta r)^{n}}{n !} \beta \int_{0}^{t-n \tau} \mathrm{e}^{-(\beta+\delta-\alpha) t^{\prime}} t^{\prime n} d t^{\prime} \\
& -s_{0} \frac{(\beta r)^{n-1}}{(n-1) !} \beta \int_{0}^{t-n \tau} \mathrm{e}^{-(\beta+\delta-\alpha) t^{\prime}} t^{\prime n-1} d t^{\prime} .
\end{aligned}
$$

The integrals may be solved by integration by parts:

$$
\begin{aligned}
I_{k}= & -\frac{1}{\beta+\delta-\alpha}\left(\mathrm{e}^{-(\beta+\delta-\alpha)(t-n \tau)}(t-n \tau)^{k}-\mathrm{e}^{-(\beta+\delta-\alpha) 0} 0^{k}\right) \\
& +\frac{k}{\beta+\delta-\alpha} I_{k-1}
\end{aligned}
$$

for $k$ either $n$ or $n-1$. If we bear in mind that the first exponent here vanishes quickly, we find that, except immediately after $n \tau$, both the first parts vanish. Of course, $I_{0}=(1-\exp [(t-n \tau)(\beta+\delta-\alpha)]) /(\beta+\delta-\alpha)$, and approximately $I_{0}=1 /(\beta+\delta-\alpha)$ and so:

$I_{k}=\frac{k !}{(\beta+\delta-\alpha)^{k+1}}$.

If we now substitute $I_{n}$ and $I_{n-1}$ :

$A(t) \approx A(n \tau)+s_{0} \frac{(\beta r)^{n-1}}{(\beta+\delta-\alpha)^{n+1}} \beta[\beta r-(\beta+\delta-\alpha)]$. 
The last term is equal to:

$s_{0} \frac{(\beta r-(\beta+\delta-\alpha))}{r(\beta+\delta-\alpha)}\left(\frac{\beta r}{\beta+\delta-\alpha}\right)^{n}$.

In other words, $A(t)$ at any time, other than during the very short spells of bursting of IRBC, is given as the sum of a geometric series. If we sum this series and multiply by $\mathrm{e}^{-\alpha t}$, we find the total parasitaemia for $t>\tau$ as :

$y(t)=\left[y(\tau)+s_{0} \beta\left(r^{n}\left(\frac{\beta}{\beta+\delta-\alpha}\right)^{n}-1\right)\right] \mathrm{e}^{-\alpha t}$

for $t>\tau$ and $y(t)=\left(\mathrm{e}^{-\alpha \mathrm{t}}-\mathrm{e}^{-(\beta+\delta) t}\right) \beta_{s_{0}} /(\beta+\delta-\alpha)$ for $0<t<\tau$ (first cycle).

Of course, our solution for $y$ is suitable only for the period after merozogony. At the short merozogony stage we would be dependent on numerical solution. The parameters for numerical simulation could be deduced from fitting the analytical solution to data.

\section{Desynchronization of wave packets}

For the genetically determined case, let us assume a wave packet with normal distribution of the peak $N(\tau, \sigma)$. Each wave reproduces itself exactly. For example, a wave which had its first peak at $47 \mathrm{~h}$ will have its tenth at $470 \mathrm{~h}$. Thus we expect a widening of the wave packet. If we assume that the standard deviation is fairly small, relative to the period (typically a few hours as opposed to $48 \mathrm{~h}$ ), the $k$ th peak will be at $k \tau$. The variance $\phi_{i}\left(k\left(\tau-\tau_{i}\right)\right)^{2}$, with $\phi_{i}$ the relative frequency of the period $\tau_{i}$. Thus the standard deviation is simply the initial standard deviation multiplied by $k: N(k \tau, k \sigma)$.

For the case of random fluctuations, each individual peak is reproduced at a time distributed $N(\tau, \sigma)$. Thus strict periodicity is not to be valid for individual waves. There is no correlation between the fluctuations from the average time between peaks $\tau$ for subsequent peaks. Thus the time at which a specific wave should recur has a standard deviation of $k^{1 / 2} \sigma$ (repeating an experiment $k$ times and summing the result). Thus the loss of synchrony in the genetically determined case is proportional to the time, while in the random case is proportional to its square root.

\section{REFERENCES}

ANDERSON, R. M., MAY, R. M. \& GUPTA, s. (1989). Nonlinear phenomena in host-parasite interactions. Parasitology 99 (Suppl.), S59-S79.

ANTIA, R., NOWAK, M. A. \& ANDERSON, R. M. (1996). Antigenic variation and the within-host dynamics of parasites. Proceedings of the National Academy of Sciences, USA 93, 985-989.

BANNISTER, L. \& DLUZEWSKi, A. (1990). The ultrastructure of red cell invasion in malaria infection: a review. Blood Cells 16, 257-292.
BOYD, M. F. \& KITCHEN, S. F. (1937). On the infectiousness of patients infected with Plasmodium vivax and Plasmodium falciparum. American Fournal of Tropical Medicine 17, 253-262.

Caillard, v., Beauté-lafitte, A., Chabaud, A. G. \& LANDAu, I. (1992). Plasmodium vinckei petteri: identification of the stages sensitive to arteether. Experimental Parasitology 75, 449-456.

Cheng, Q., LaWrence, G., ReEd, C., Stowers, A., RANFORD-CARTWRight, L., CREASEy, A., CARTER, R. \& SAUl, A. (1997). Measurement of Plasmodium falciparum growth rates in vivo: a test of malaria vaccines. American Fournal of Tropical Medicine and Hygiene 57, 495-500.

DEHARO, E. (1995). Etude des mecanismes regissant le rythme des schizognies erythrocytaires des Plasmodium. Nouvelles techniques, nouvelles donnees, These de doctorat, Université du Droit et de la Sante de Lille.

DRUILHE, P. \& PERIGNON, J. L. (1994). Mechanisms of defense against $P$. falciparum asexual blood stages in humans. Immunology Letters 41, 115-120.

GRAVENOR, M. B. \& KWIATKOWSKI, D. (1998a). An analysis of the temperature effects of fever on the intra-host population dynamics of Plasmodium falciparum. Parasitology 117, 97-105.

GRAVENOR, M. B. \& LLOYD, A. L. (1998b). Reply to: Models for the in-host dynamics of malaria revisited: errors in some basic models lead to large overestimates of growth rates. Parasitology 117, 409-410.

GUPTA, S., HILl, A. V., KWIATKOWSKI, D., GREENWOOD, A. M., GREENWOOD, B. M. \& DAY, K. P. (1994). Parasite virulence and disease patterns in Plasmodium falciparum malaria. Proceedings of the National Academy of Sciences, USA 91, 3715-3719.

KWIatKowski, D. \& NOWAK, M. (1991). Periodic and chaotic host parasite interactions in human malaria. Proceedings of the National Academy of Sciences, USA 88, 5111-5113.

MCKenZie, F. E. \& BOSSERT, W. H. (1997). The dynamics of Plasmodium falciparum blood-stage infection. Fournal of Theoretical Biology 188, 127-140.

MOLinEaUX, L. \& DiETz, K. (1999). Review of intra-host models of malaria. Parassitologia 41, 221-231.

SAUL, A. (1998). Models for the in-host dynamics of malaria revisited: errors in some basic models lead to large over-estimates of growth rates. Parasitology 117, 405-407.

SIllamut, K., PHU, N. H., WhitTy, C., TURNER, G. D., LOUWRIER, K., MAI, N. T., SIMPSON, J. A., HIEN, T. T. \& White, N. J. (1999). A quantitative analysis of the microvascular sequestration of malaria parasites in the human brain. American Fournal of Pathology 155, 395-410.

White, N. J. (1997). Assessment of the pharmacodynamic properties of antimalarial drugs in vivo. Antimicrobial Agents and Chemotherapy 41, 1413-1422.

White, N. J., Chapman, D. \& WatT, G. (1992). The effects of multiplication and synchronicity on the vascular distribution of parasites in falciparum malaria. Transactions of the Royal Society of Tropical Medicine and Hygiene 86, 590-597. 